\title{
IPv6 address autoconfiguration in geonetworking- enabled VANETs: characterization and evaluation of the ETSI solution
}

\author{
Marco Gramaglia ${ }^{1,2^{*}}$, Carlos J Bernardos ${ }^{2}$, Ignacio Soto ${ }^{3}$, Maria Calderon $^{2}$ and Roberto Baldessari ${ }^{4}$
}

\begin{abstract}
In this article we make a thorough characterization and evaluation of the solution standardized by the European Telecommunications Standards Institute for IPv6 transmission of packets over geographical location aware vehicular networks. In particular, we focus on IPv6 address auto-configuration, one of the required pieces to enable Internet connectivity from vehicles. Communications in vehicular networks are strongly dependent on the availability of multi-hop connectivity to the fixed infrastructure, so also we analyze the probability of achieving this connectivity under different circumstances, and we use the results to identify interesting target scenarios for address autoconfiguration mechanisms. Keeping those scenarios in mind, we perform a characterization and deep evaluationanalytically and by means of simulations-of the standardized IPv6 address au-toconfiguration solution; proposing some configuration guidelines and highlighting the scenarios where complementary enhancements might be needed.
\end{abstract}

Keywords: VANETs, geonetworking, IP address auto-configuration, intelligent, transportation systems, cooperative systems, ETSI

\section{Introduction}

Vehicular networks architectures typically allow for two types of communications: vehicle-to-vehicle $(\mathrm{V} 2 \mathrm{~V})$ and infrastructure-to-vehicle (I2V). V2V communications are mainly used by safety applications (e.g., cooperative collision warning, pre-crash sensing/warning, hazardous location, cooperative awareness) while I2V communications are typically used by traffic efficiency applications (e.g., traffic Signal Phase and Timing-SPAT, recommended speed and route guidance). There is, however, increasing interest in also supporting Internet communications from and to vehicles. By allowing classical and new IP services to be accessible from vehicles, users would see an additional benefit in the installation of a communication system in their cars, and this would help increasing user acceptance and in turn facilitate initial deployment and market penetration.

Car manufacturers as well as public authorities are working together for the definition of communications

\footnotetext{
*Correspondence: marco.gramaglia@imdea.org

${ }^{1}$ Institute IMDEA Networks, Madrid, Spain

Full list of author information is available at the end of the article
}

standards in the vehicular environment. Because of the growing interest, vehicular networking has become a hot research topic in the last few years, due to its potential applicability to increase road safety and driving comfort. In particular, the use of vehicular ad hoc networks (VANETs) is being considered as the base candidate technology for these cooperative systems that are expected to significantly reduce the number of traffic accidents, improve the efficiency and comfort of road transport, and also enhance the passengers' communications experience. Although many applications of vehicular communications were already identified in the $80 \mathrm{~s}$, large-scale deployment of such systems has finally become possible due to the availability of new technologies, such as devices based on the IEEE 802.11 standard family, which seem to offer an affordable compromise between performance and system complexity. The primary advantage of deploying this kind of self-organized network is the fact that timely critical applications, such as safety-of-life applications, can be implemented by letting vehicles directly communicate to each other, instead of relying on centralized entities. 
Working groups and standardization bodies such as IEEE 1609, ${ }^{\mathrm{a}}$ ISO TC204, ${ }^{\mathrm{b}}$ ETSI TC ITS $^{\mathrm{c}}$ and the Car-toCar Communications Consortium ${ }^{\mathrm{d}}$ (C2C-CC) have been working on providing vehicles with connectivity, both among them and to the Internet. So far, priority has been given to those capabilities required to enable safety applications, but there is an increasing interest in also enabling Internet access from the vehicles. The Internet connectivity capability is seen by consumers as a very valuable feature in a mobile phone, a television, or any electronic equipment- and thus has an impact on the user's decision when choosing what to buy-and so is expected to be the case in the near future for cars. The deployment effort required to equip roads segments with wireless attachment points connected to a network infrastructure is often regarded as a major obstacle. The use of multi-hop networks considerably aids in reducing this difficulty, as the density of needed access points is reduced. This, however, brings the challenge of how to smoothly interconnect vehicular networks to the Internet.

The European Telecommunications Standards Institute (ETSI) TC ITS is the technical committee that received a standardization mandate from the European Commission for the development of short-range Intelligent Transport Systems (ITS) communication protocols. Recently, the ETSI has finalized the standardization of the mechanisms [1] required to integrate IPv6 in the harmonized communication system for European ITS [2]. The ETSI TC ITS architecture benefits from geographical location awareness of cars (it is assumed that all vehicles know its geographical location, by means of using a GPS or similar device) to extend the concept of IPv6 link to a specific geographical area. This article first presents the geographical location aware vehicular architecture standardized by ETSI, commonly referred to as geonetworking, and then describes in detail how IPv6 datagram transmission and standard IPv6 stateless address autoconfiguration mechanisms are performed on top of the ETSI geonetworking protocol stack (Section 2). Since the ETSI geonetwork-ing architecture is based on the use of a vehicular ad hoc network (VANET), we identify in this article the range of conditions in which multi-hop connectivity to the Internet from vehicles is effective, considering the vehicular density, the coverage radius of the wireless technology, and the distance between attachment points in the infrastructure (Section 3). These conditions define the main target scenarios in which vehicles can use IPv6 to communicate, and therefore the scenarios in which address auto-configuration mechanisms for vehicular networks must work. Next, the article includes a rigorous analysis of the ETSI stateless IPv6 address autoconfiguration mechanism, based on the identified target scenarios. An analytical model (Section 4) and a simulation-based evaluation of its performance are provided, which helps us derive configuration guidelines of the solution depending on the scenario where it is deployed and the traffic conditions (Section 5). Finally, we conclude the paper by summarizing the main conclusions of our in-depth analysis, highlighting the situations in which additional extensions to the base solution defined by ETSI may be required, and briefly discussing the associated trade-offs (Section 6).

\section{Background}

\subsection{Connecting VANETs to the internet}

In order to connect VANETs to the Internet, vehicles have to be provided with a full Internet Protocol (IP) stack, as IP is the basic building block for Internet communications. IPv6 has been adopted as the version of the IP protocol by all the previously mentioned standardization bodies and consortia and has been included in their communication architectures. We can identify three main functionalities required to bring IP into the vehicular networks: (a) the capability of vehicles to autoconfigure an IP address, (b) IP mobility mechanisms suited for vehicular scenarios, and (c) mechanisms for an efficient transmission and forwarding of IP datagrams within the vehicular network. In this paper we focus on the first topic, that is the auto-configuration of IP addresses by nodes of a VANET. IPv6 provides some standardized mechanisms of IP address auto-configuration, both stateless $[3,4]$ and stateful [5] that cannot-or at the very least are hard to-be applied without any modification in vehicular environments. The main causes of this fact are the multi-hop nature of VANETs and their lack of a single multicast-capable link for signaling, which prevent current IP address auto-configuration-related protocol specifications from being used as is in VANETs. Therefore, a key research issue is how to auto-configure IPv6 addresses in a VANET. The same problem occurs in general in any unmanaged multi-hop network. Among these, Mobile Ad hoc Networks (MANETs) have received a remarkable attention in the research area for years, and there even exists a working group in the IETF, ${ }^{\text {e }}$ called AUTO-CONF, that is chartered to work on the standardization of an address auto-configuration solution for MANETs [6].

Two main approaches that can be followed to integrate IP in a multi-hop vehicular network:

1. Making the IP layer fully aware of the multi-hop nature of VANETs. In this case, the VANET can be defined as a set of IP routers that are interconnected by a multitude of IP links. The high dynamics of each individual link strongly contributes to the overall addressing and routing management overhead. In particular, in order to understand this complexity, we recall the 
assumption underpinning IP routing, which requires IP addresses assigned to nodes terminating different links to belong to non-overlapping prefixes.

Two IP prefixes p::/1_p and q::/l_q are non-overlapping if and only if there is no IP address p::a/l_p configured from $\mathrm{p}:: / \mathrm{l} \_\mathrm{p}$ that also belongs to $\mathrm{q}:: / \mathrm{l} \_\mathrm{q}$, and vice versa. ${ }^{\mathrm{f}}$ In order to enable IP routing, an overwhelming amount of short-lived routes is required, posing extremely challenging management issues.

An example of solution that falls in this category and is particularly designed for VANET environments is the Vehicular Address Autoconfigura-tion (VAC) solution, proposed by Fazio et al. in [7]. This solution exploits the VANETs topology and an enhanced DHCP service with dynamically elected leaders to provide a fast and reliable IP address configuration. VAC organizes leaders in a connected chain such that every node (vehicle) lies in the communication range of at least one leader. This hierarchical organization allows limiting the signaling overhead for the address management tasks. Only leaders communicate with each other to maintain updated information on configured addresses in the network. Leaders act as servers of a distributed DHCP protocol and normal nodes ask leaders for a valid IP address whenever they need to be configured. The main drawbacks of this solution are the assumption of linear topology and group movement which limits the applicability scope, the overhead due to the explicit management signaling (e.g., between leaders), and the possible security threat due to the critical tasks carried out by the leaders. Some of the solutions proposed for Mobile Ad Hoc Networks (MANETs) [6] may also be used for vehicular networks. Most of these solutions and VAC share the problem that they require modifications to the IP stack of the nodes, as they do not rely on existing standardized IPv6 address auto-configuration solutions.

2. Hiding the multi-hop nature of VANETs from the IP layer. In this approach, the concept of IPv6 link is extended to a set of nodes which might not be directly reachable within one physical hop. A protocol located below IP presents a flat network topology, ensuring that the link seen by the IP layer includes all the nodes of the extended set, even those that are not directly reachable. In this case, existing IP address auto-configuration mechanisms could be used with minor modificationsand even without any.

This last approach was followed by the European GeoNet project, ${ }^{\mathrm{g}}$ which contributed to the solution finally standardized by ETSI. Two similar solutions have been proposed:(a) Geographically Scoped stateless Address Configuration (GeoSAC) [8], initially proposed before GeoNet started, and further developed during the lifetime of the project; and (b) [9], that adopts this same concept but has many and essential differences in the realization. The latter solution does not assure compatibility with legacy IPv6 protocol implementations and requires the IPv6 protocol to be geo-aware.

In this paper we focus on the solution adopted by the ETSI TC ITS, which follows the second approach, hiding the multi-hop nature of the VANET from the IP layer. We next present this system architecture and define the terms used in the rest of the paper.

\subsection{ETSI TC ITS IPv6 integration system architecture}

ETSI TC ITS is developing a set of protocols and algorithms that define an harmonized communication system for European ITS applications taking into account industry requirements like in particular those coming from the Car-to-Car Communications Consortium. In the ETSI TC ITS network architecture [2], vehicles are equipped with devices called Communication and Control Units (CCUs), which implement the ETSI protocol stack (see Figure 1, in which only the part of the stack involved in IPv6 communications is shown). Vehicles can communicate with each other or with fixed roadside ITS stations (also called Roadside Units, RSUs) installed along roads. CCUs and RSUs implement the same network layer functionalities and form a self-organizing network. RSUs can be connected to a network infrastructure, most likely an IP-based network. On-board application hosts including passenger devices attached to the vehicle on-board system are called Application Units (AUs). Passenger devices are assumed to have a standard IPv6 protocol stack, whereas CCUs act as gateways for the in-vehicle network optionally enhanced with the Network Mobility Basic Support protocol [10].

The ETSI GeoNetworking (GN) protocol [11], currently under completion and expected to be published soon, plays the role of a sub-IP layer, offering a flat network view to the IPv6 layer and dealing with the multihop routing within the VANET (nodes within the same area-i.e., attached to the same IP link- might not be directly reachable, but are portrayed as such by the subIP layer). The ETSI has standardized a protocol adaptation sub-layer referred to as the GN6ASL (GeoNetworking to IPv6 Adaptation SubLayer) [1] which allows for the transport of IPv6 packets by ETSI GeoNetworking protocol, enabling sub-IP multi-hop delivery of IPv6 packets. The ETSI GN geo-broadcasting capability is used by the GN6ASL in order to shape link-local multicast messages to geographical areas.

Figure 2 shows the subset of the ETSI TC ITS system which is relevant to understand how IPv6 is integrated in the ETSI geonetworking architecture and the way the ETSI GN layer is used to logically create links-called Geographical Virtual Links (GVLs) - mapped to areascalled GVL Areas. We will explain this in more detail in Section 2.3. We want to highlight here how IP packets 


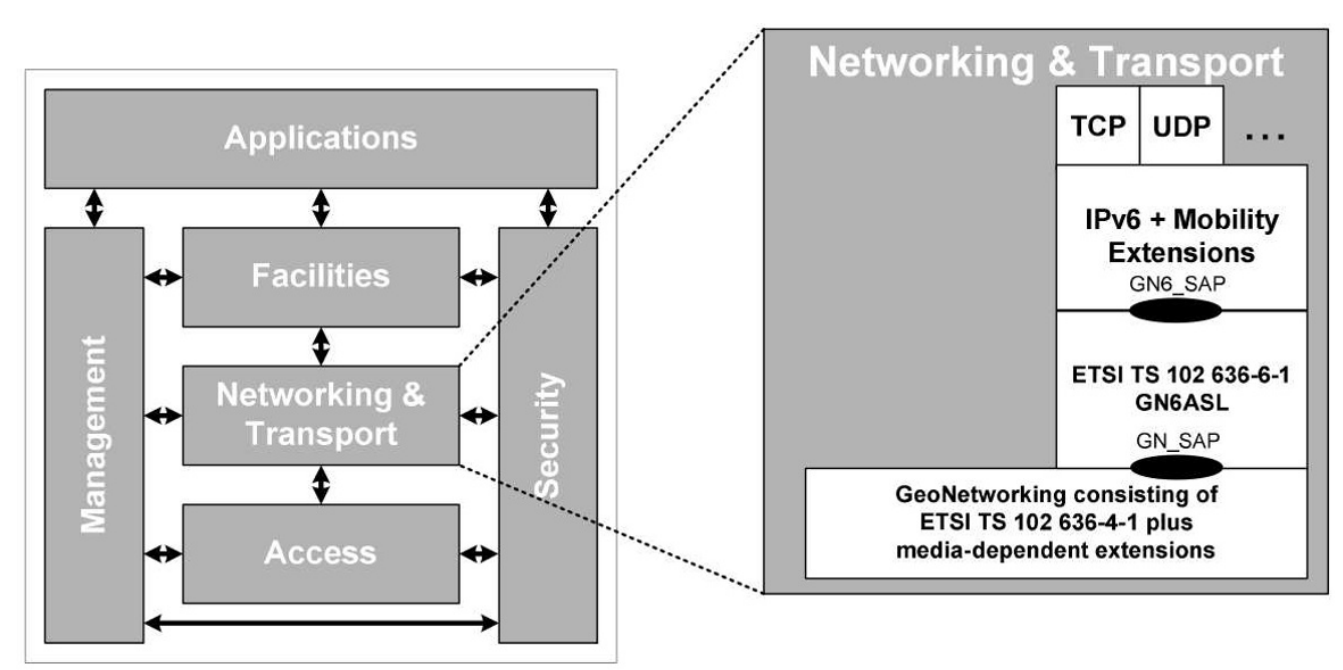

Figure $1 \mathrm{GN} 6 \mathrm{ASL}$ in the ITS station architecture

are sent in the system, using the scenario depicted in Figure 2. Let us suppose a device within Vehicle C wants to communicate with a node in the Internet. For that communication to happen, the Vehicle $\mathrm{C}$ has to send packets to the RSU of its area-that is the next hop at the IP layer-and this requires at the ETSI GN layer Vehicle $C$ to send packets to Vehicle B, which forwards them to Vehicle A, that finally delivers them to the RSU. Note that this multi-hop forwarding is required because Vehicle $C$ is not within the radio coverage of the RSU. This example shows that in a system architecture based on short-range communication devices, the effective provisioning of Internet-based applications over multi-hop communication strongly depends on mobility. Single-hop vehicular Internet access based on WLAN has already been investigated in highway scenarios [12], concluding that the link between CCU and RSU is stable enough to allow for several types of applications. When considering multi-hop communication, the applicability scope of Internet-based applications might need to be reduced to lower speed scenarios (e.g., urban or semi-urban), to a proper ratio of CCUs per installed
RSU and to a realistic maximum number of hops (to be determined). Section 3 addresses these particular issues, assessing under which conditions it is realistically feasible to support IP unicast multi-hop communications.

\subsection{IPv6 stateless address configuration over the ETSI TC ITS architecture}

The ETSI specification devoted to the integration of IPv6 and the geonetworking architecture not only describes how IPv6 packets are exchanged between ITS stations and how the GN6ASL is presented to the IPv6 layer as a link-layer protocol, but also explains how IPv6 addresses can be automatically configured by ITS stations, namely CCUs. The specification [1] only considers the use of stateless address autocon-figuration schemes, as stateful ones present higher latencies (due to the several round-trip time signaling messages) and requires of greater management effort. Manual configuration is also not recommended.

The ETSI solution is based on the Geographically Scoped stateless Address Configuration (GeoSAC) solution [8], which can be considered as one particular

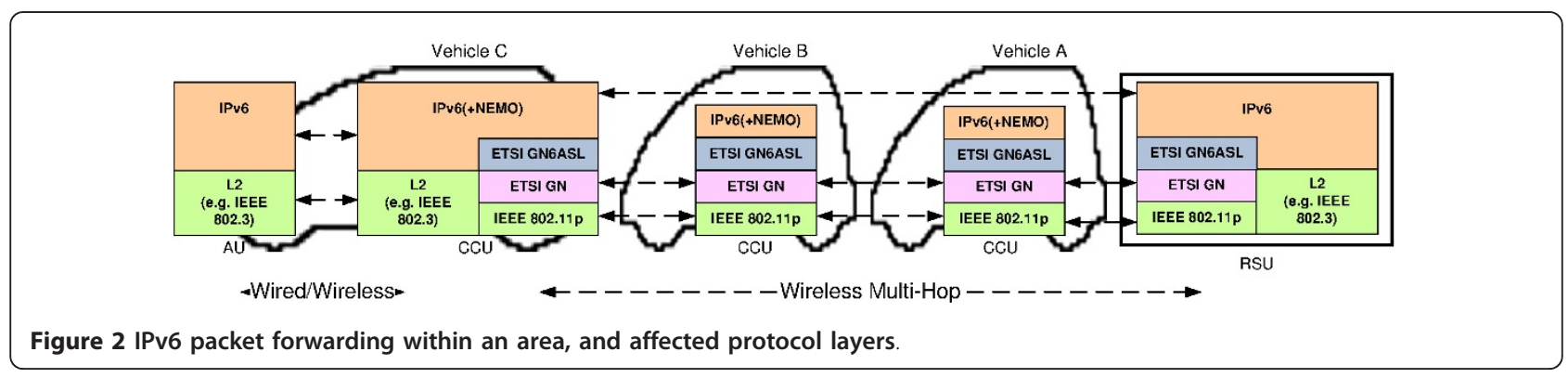


realization of the ETSI standardized mechanism. In the rest of the paper we refer to the ETSI IPv6 address stateless autoconfiguration solution as ETSI SLAAC.

ETSI SLAAC adapts the standard IPv6 SLAAC (Stateless Address Auto-Configuration) mechanism so it can be used in multi-hop vehicular ad hoc networks, by taking advantage of the geographical location awareness capabilities of the vehicles. In ETSI SLAAC, the concept of IPv6 link is extended to a well-defined geographical area (i.e., GVL area) associated with a point of attachment to an infrastructure-based network that plays the role of the IPv6 Access Router (AR).

The GeoNetworking-IPv6 Adaptation Sub-Layer (GN6ASL) (see Figure 1) is a sub-IP layer sitting on top of the ETSI GN layer. The ETSI GN layer deals with ad hoc routing by using geographic location information, while the GN6ASL presents to the IPv6 layer a flat network topology. Consequently, the link seen by the IPv6 layer includes nodes that are not directly reachable but are portrayed as such by the sub-IP layer (see Figure 3). This layer provides IPv6 with a link-local multicast-capable link, the Geographical Virtual Link (GVL), which includes a non-overlapping partition of the VANET formed by all nodes within a certain geographical area (the GVL area).
Each GVL area is managed by at least one RSU that acts as an IPv6 Access Router and sends standard IPv6 Router Advertisements (RA), carrying the IPv6 prefix(es) inside the Prefix Information Option (PIO). Nodes receiving the RAs can then build a valid IPv6 address out of the included IPv6 prefix, following the standard SLAAC mechanism, i.e., the host generates an address by joining the prefix received from the RA and the network identifier derived by its MAC address.

The link-local multicast capability emulation is achieved by relying on the geo-multicast/geo-broadcast capabilities provided by the ETSI GN layer. In particular, in order to be link-local multicast capable, an IP link must provide symmetric reachability [3], which is normally not accomplished by virtual links spanning multiple physical links due to the lack of reference boundaries. Link-local multicast packets are forwarded with geographical knowledge, so that a node processes a packet only if it was addressed to the area where the node is located. The geographic scoping provides nonvariable virtual link boundaries which enable symmetric reachability. For RAs, this means that RAs must be delivered to-and only to-the nodes that are part of the same IPv6 link, nodes that are actually connected via multiple wireless hops. If a multi-hop path exists, all the

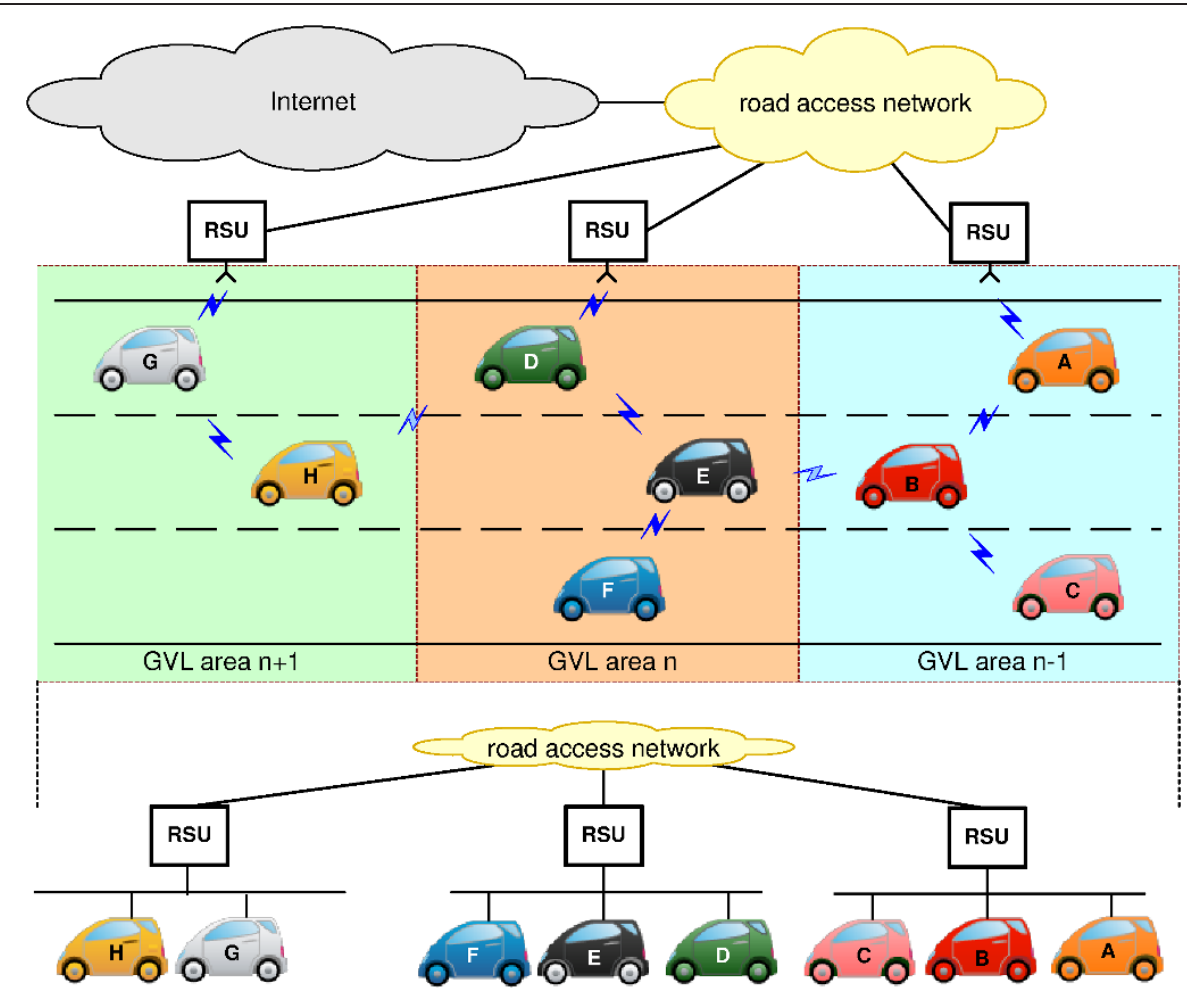

Figure 3 Geographical area partitioning and IPv6 virtual link abstraction. 
nodes within the area will receive a copy of the RA, and the IPv6 instance running above the geonetworking will process the message as if the node was directly connected to the access router that issued the message.

It is assumed that MAC addresses (or a different identifier that can be used for IPv6 address generation purposes) of vehicles are unique, at least within macroregions where vehicles are sold and can potentially communicate with each other (e.g., a continent). This property in fact is highly desirable for security and liability reasons, as it would allow (i) forensic teams to rely on vehicular communications to reconstruct accident scenes or other critical situations and, (ii) to detect malicious nodes and reduce considerably the effects of network attacks. Despite uniqueness of identifiers, privacy of users can be protected by equipping vehicles with sets of unique identifiers to be used for limited intervals as pseudonyms [13].

These identifiers could be assigned by authorities and, when coupled with the usage of digital certificates and cryptographic protection [14], this mechanism can accomplish support for liability as well as privacy protection from malicious users (commonly referred to as revocable privacy). Assuming that the IPv6 prefix announced by the RSU is exclusively assigned to this area, the address uniqueness is verified, and therefore no Duplicate Address Detection (DAD) mechanism is required. Note that the proposed solution could be applied to multiple RSUs acting as bridges connected to one single Access Router. This might be a good deployment choice in scenarios where single-hop connectivity to the infrastructure is preferred while it is also required to reduce the number of IPv6 address changes (e.g., city environment).

A technique that maximizes the benefits of ETSI SLAAC consists in shaping the GVL areas assigned to the RSU in a adjacent and logically non-overlapping fashion, as depicted in Figure 3. By doing so, the following key advantages are obtained: (i) unequivocal gateway selection is achieved with the infrastructure having full control on it, ${ }^{\mathrm{h}}$ as only one RSU is assigned per geographical area; (ii) a network partitioning is obtained that supports movement detection procedures of IPv6 mobility and also allows for location-based services. In particular, a vehicle moving across regions served by different Access Routers experiences a sharp sub-net change, without traversing gray areas where Router Advertisements are received from multiple access points (potentially leading to ping-pong effects).

Before characterizing and analyzing the performance of the ETSI SLAAC solution, we next analyze under which conditions it is realistically feasible to support IP unicast multi-hop communications in a vehicular environment.

\section{Effectiveness of vehicular multi-hop communications}

Vehicular networks using short-range wireless technologies, such as IEEE 802.11-based ones, rely on multi-hop communications to extend the effective coverage of the RSUs deployed on the roadside. One of the main challenges that VANETs pose is the minimum degree of technology penetration that is needed in order to ensure that there is enough density of communication-enabled vehicles to support multi-hop connectivity between the intended peers (e.g., for the case of Internet communications, between the vehicle and the RSU). This problem becomes even more problematic during the time of the day when roads are less busy. In these environments, communications can become difficult because radio devices often operate at their design limits (large distances, multi-path signal propagation, critical packet length vs. channel coherence time ratio, etc.), which amplifies the effect of layer-2 inefficiencies due to hidden node scenarios. Furthermore, the probability of having a multi-hop path between two nodes is lower in sparse scenarios. On the other hand, when roads become more crowded, speeds are lower, links are more reliable, and the chances for two arbitrary nodes to be connected by at least one stable multi-hop path are higher.

Deploying vehicular networks without dead zones (i.e., areas not served by any RSU) is economically inefficient in non-urban locations. As we have mentioned above, in the ETSI TC ITS architecture, vehicles form a self-organized multi-hop network. This multi-hop network is used to forward packets between the RSU and the CCUs within the RSU's area of influence (i.e., associated GVL area), and therefore extends the effective coverage area of the RSU. In order to assess the feasibility of vehicular communications in practical scenarios, it is necessary to evaluate whether wireless multi-hop communications are possible in different vehicular situations. To do so, we model and analyze the probability of having a multi-hop path between a sender and a receiver, studying the impact of different parameters, such as vehicular speed and density, wireless radio coverage, etc. We present our mathematical model first and then validate it via simulations.

Given two nodes separated by a distance $S, P_{\mathrm{mhc}}(S)$ is the probability of having multi-hop connectivity (mhc) or, in other words, the probability that one chain of inter-connected vehicles between the two nodes exists. This probability depends-as we show below-on the distance between the two nodes, the radio coverage, and the vehicular density. Figure 4 shows an example of a chain of interconnected vehicles between a car and an RSU.

We model the distance $D$ between consecutive vehicles (inter-vehicle spacing) as exponentially distributed 


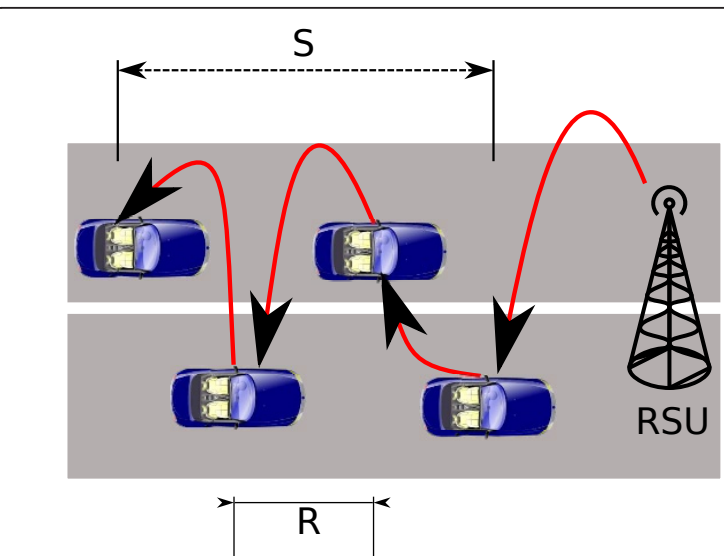

Figure 4 Multi-hop connectivity between a car and an RSU.

$[15,16]$, with parameter $\beta$, with its Probability Density Function (PDF) given by:

$$
f_{D}(d)=\beta e^{-\beta d}, \quad d \geq 0,
$$

where $\beta$ is the vehicular density. Let $R$ be the wireless coverage radius. The distance between two consecutive vehicles that are part of a connected multi-hop chain of vehicles (the inter-vehicle gap is smaller than $\mathrm{R}$ ) follows a truncated exponential distribution [17]:

$$
f_{t e}(d)= \begin{cases}\frac{\beta e^{-\beta d}}{1-\mathrm{e}-\beta R}, & 0<d<R, \\ 0, & \text { otherwise. }\end{cases}
$$

The length of a multi-hop connected chain of $n+1$ vehicles $(Y)$ can be represented as the sum of $n$ independent exponential truncated variables. The PDF of $Y$ can be obtained by the method of characteristic functions [17]:

$$
\begin{aligned}
g_{Y}(\gamma ; n)= & \frac{(\beta b)^{n}}{(n-1) !} e^{-\beta_{y}} \sum_{k=0}^{k_{0}}(-1)^{k}\left(\begin{array}{l}
n \\
k
\end{array}\right)(y-k R)^{n-1} ; \\
& k_{0} R<\gamma<\left(k_{0}+1\right) R
\end{aligned}
$$

where $k_{0}=0,1, \ldots, n-1$, and $b=\left(1-\mathrm{e}^{-\beta R}\right)$.

Let $a=\left(k_{0}^{\prime}+c\right) R$, where $k_{0}^{\prime}$ is an integer, and $0 \leq c<1$. The Cumulative Distribution Function (CDF) of $Y$ evaluated at $a$ is $G_{Y}(a ; n)=\int_{0}^{a} g_{Y}(y ; n) \mathrm{d} y$ :

$$
\begin{aligned}
G_{Y}(a ; n)= & \frac{1}{\left(1-\mathrm{e}^{-\beta R}\right)^{-n}} \sum_{k=0}^{k_{0}}(-1)^{k}\left(\begin{array}{l}
n \\
k
\end{array}\right) \mathrm{e}^{-\beta k R} \\
& Q\left[2\left(k^{\prime}{ }_{0}-k+c\right) R \beta, 2 n\right] .
\end{aligned}
$$

where $Q[u, w]=P\left(\chi^{2}(w)<u\right)$ and $\chi^{2}(w)$ is a chisquare variable with $w$ degrees of freedom. Since the probability $P(i)$ of having a connected chain of $i$ hops is given by $\left(1-\mathrm{e}^{-\beta R}\right) \mathrm{e}^{-\beta R}$, the PDF and CDF of the length $(L)$ of a connected multi-hop chain of vehicles can be derived using the law of total probability:

$$
\begin{aligned}
f_{L}(l) & =\sum_{i=0}^{\infty} P(i \text { hops }) g_{Y}(l ; i) \\
& =\sum_{i=0}^{\infty}\left(1-e^{-\beta R}\right)^{i} e^{-\beta R} g_{Y}(l ; i), \\
F_{L}(l) & =P_{L}(L \leq l) \int_{0}^{l} f_{L}(u) \mathrm{d} u \\
& =\sum_{i=0}^{\infty}\left(1-e^{-\beta R}\right)^{i} e^{-\beta R} G_{Y}(l ; i) .
\end{aligned}
$$

Based on this, $P_{\mathrm{mhc}}(S)$ is given by:

$$
P_{\text {mhc }}(S)=1-F_{L}(S) \text {. }
$$

Another factor that should be considered to assess the feasibility of vehicular multi-hop communications is the number of available lanes in a road. Our previous analysis is valid regardless of the number of lanes, thanks to the properties of the exponential distribution. If we consider several lanes, and in each one we model the spacing between cars by an exponential distribution, not necessarily with the same mean (the different lanes can have different car densities), the resulting space between cars in the road (not matter in which lane) is exponentially distributed with mean the average of the means in each lane. Therefore, we do not assume any particular number of lanes throughout the rest of the paper, unless indicated explicitly. Note that we are approximating the car distribution assuming that there is no correlation between the lane geometry and the car distribution. This means that we disregard the spatial correlation introduced by traffic regulation and congestion. The consequences of this assumption are evaluated in the next section.

In order to validate our analysis of $P_{\mathrm{mhc}}$, we performed a large amount of experiments via simulation under different traffic conditions. The simulator ${ }^{i}$ was developed using Matlab and it implements the scenario described in this section, namely vehicles distributed in a one-dimensional road, traveling at a pre-defined and constant speed, with an exponential inter-vehicular distance and a maximum wireless radio coverage, assuming an ideal wireless technology (no packet losses nor collisions and infinite bandwidth). Although the simulator does not consider a real wireless model, we argue that it is enough to show the correctness of our mathematical model, as it fully implements the behavior we are modeling. Obtained results show that our mathematical 
analysis perfectly models the probability of having multihop connectivity (assuming the aforementioned simplifications). We do not show these validation results due to space constraints. Simulation and experimental results are shown in Section 5, where we use a more advanced simulator $(\mathrm{OMNeT++})$ that does include a complete wireless model to validate our formulation of the configuration time of the ETSI SLAAC solution.

In the following, we focus on analyzing the scenarios in which unicast communications using a multi-hop vehicular network are feasible. There are three parameters that have an impact on the probability of having multi-hop connectivity between two nodes:

- The distance $S$ between the nodes. The larger this distance is, the lower is the probability of having connectivity. If we focus on the vehicle-to-Internet scenario, this value would be related to the distance between a moving vehicle and the fixed RSU, and therefore it depends on how RSUs are deployed.

- The vehicular density $\beta$. The probability of having connectivity increases with the vehicular density. The density depends on the traffic conditions (i.e., the time of the day and road) and the type of road (i.e., there are roads more congested than others). Vehicles density and speed are usually correlated as well, since the minimum safety distance between vehicles depends on the speed [18].

- The wireless coverage radius $R$. The effective radius depends on the specific wireless access technology, the transmission power at the antenna, the antenna radiation pattern, and the instantaneous channel response. The probability of having multi-hop connectivity is obviously very much affected by $R$, shorter values leading to lower probabilities.

If we fix the value of $R$, which is equivalent to assuming a reference system, it is interesting to study which is the minimum vehicular density required to ensure a certain probability of multi-hop connectivity between two nodes, depending on their distance. Figure 5 depicts the simulation results obtained for three different values of $R(150,300$, and $450 \mathrm{~m})$, which represent a realistic range of wireless coverage radius for wireless access technologies expected to be used in vehicular communications [19]. The results are plotted in three dimensions, so it can be observed how the vehicular density $\beta$ and the distance $S$ between the two nodes affect the multihop connectivity probability. An horizontal plane for $P_{\text {mhc }}=0.9$ is also depicted in the figures, so we can observe which are the combinations of $\beta$ and $S$ that result in values of $P_{\text {mhc }}$ higher than $90 \%$. The cut (intersection) of horizontal planes corresponding to probabilities of $0.7,0.8,0.9$, and 0.95 and the 3D curve are shown in Figure 6. Using this figure we can find out which is the minimum vehicular density required to achieve a minimum multi-hop connectivity probability between two nodes separated by a given distance.

Let us take for example the reference value of $S=$ $1,000 \mathrm{~m}$. From the results in Figure 6, we can conclude that if the coverage radius $R$ is $150 \mathrm{~m}$, a vehicular density of approximately $35 \mathrm{veh} / \mathrm{km}$ or higher ensures that there is multi-hop connectivity in the $90 \%$ of the cases. Similarly, $15 \mathrm{veh} / \mathrm{km}$ are enough if $R$ is $300 \mathrm{~m}$, and 8 $\mathrm{veh} / \mathrm{km}$ for $R=450 \mathrm{~m}$. It is important to highlight that these densities are quite low and that, therefore, are likely to be found in realistic scenarios with typical traffic conditions.

In order to limit the number of results presented in the paper, we selected the following three scenarios which mostly cover a wide spectrum of potential traffic scenarios:

- Urban road: high vehicular density $(\beta=80 \mathrm{veh} / \mathrm{km})$ and low speed $(v=50 \mathrm{~km} / \mathrm{h})$.

- City highway: moderate vehicular density $(\beta=50$ veh $/ \mathrm{km})$ and moderate speed $(v=80 \mathrm{~km} / \mathrm{h})$.

- Motorway: low vehicular density $(\beta=35 \mathrm{veh} / \mathrm{km})$ and high speed $(v=120 \mathrm{~km} / \mathrm{h})$.

As it can be observed from Figure 6, it is perfectly feasible to have multi-hop connectivity in these three scenarios for most of the potential deployments (i.e., interRSU distances).

The probability of multi-hop connectivity is not the only parameter that should be considered when assessing the feasibility of vehicular communications, as the number of hops also plays an important role (i.e., the larger this number is, the lower are throughput and reliability). Figure 7 shows the average, minimum, and maximum values of the number of traversed hops (only for those communications that can take place, i.e., where a multi-hop chain of vehicles exists) for the same scenarios. From these results we can also conclude that it is not efficient from a performance viewpoint to deploy RSUs which are separated by large distances, as the number of hops would get too high, impacting the performance of the communications. It should be noted that vehicles are expected to be equipped with one single wireless radio interface for multi-hop communications using a self-configured VANETj and therefore the effective throughput decreases with the number of traversed wireless hops in the VANET.

\section{Analytical characterization of the ETSI SLAAC's performance}

The main purpose of an IP address auto-configuration protocol is to provide each node with a valid IP address as soon as possible. In the followings we derive an analytical expression of the time required by the ETSI SLAAC solution to configure an address. 


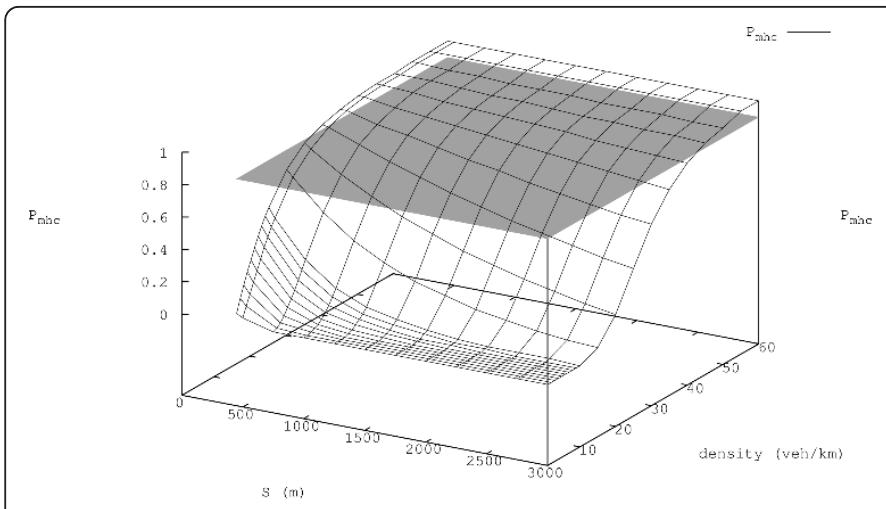

(a) $R=150 \mathrm{~m}$

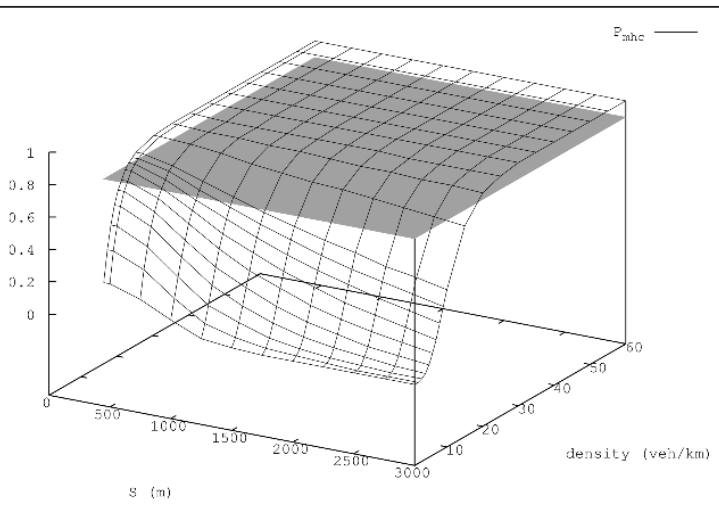

(b) $R=300 \mathrm{~m}$

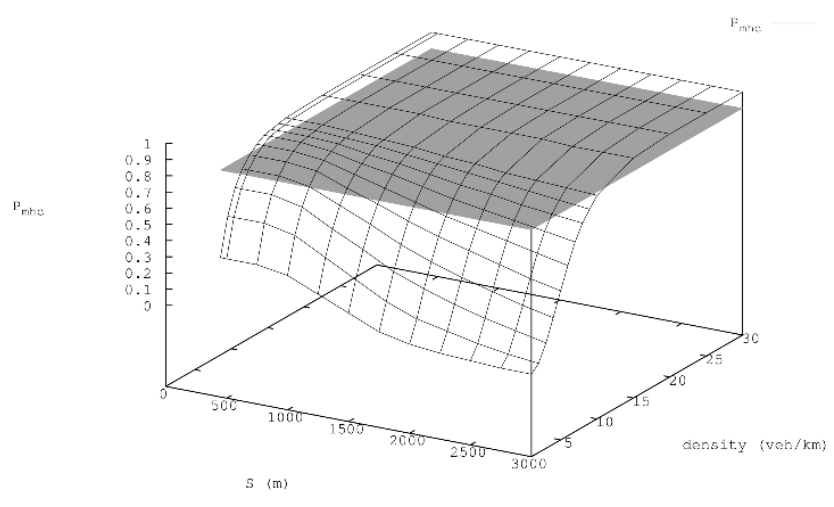

(c) $R=450 m$

Figure $5 P_{\text {mhc }}$ : simulation results (cut in $90 \%$ probability)

The address configuration time ( $\left.T_{\text {conf }}\right)$ is the time elapsed since a vehicle enters a new geographical area (therefore loosing the connectivity to the old RSU) till the moment in which it can start using the newly configured global IPv6 address. This time depends on several factors, such as the shape and size of the areas, the configuration of the RSUs and ARs, etc.

We consider that the time between two consecutive RAs sent by an RSU (or an AR in case the RSU is working in bridge mode) follows a uniform distribution between a minimum value (MnRtr AdvInterval) and a maximum value (MaxRtr AdvInterval), which we refer to as $R_{m}$ and $R_{M}$, respectively [20].

We use the following additional terminology. Let $D_{\text {rsu be }}$ the distance between two adjacent RSUs, $R$ the wireless communication range, $\beta$ the vehicular density and $v$ the speed of the vehicles. ${ }^{\mathrm{k}}$

In order to obtain a mathematical expression for

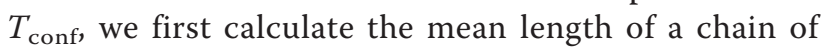
vehicles. Based on that, we are able to find out which is-on average-the length of the gap between the multi-hop chain of vehicles from the unconfigured vehicle and the RSU wireless coverage area $\bar{D}_{g a p}$ (see Figure 8).

The average distance between two consecutive vehicles can be calculated using Equation (2), and it is given by:

$$
\bar{D}=\int_{-\infty}^{\infty} d f_{t e}(d) \mathrm{d} d=\frac{1+\beta R-e^{\beta R}}{\beta\left(1-e^{\beta R}\right)} .
$$

The average length of a chain composed by $i+1$ vehicles is:

$$
\bar{L}_{\text {chain }}(i+1)=i \bar{D} \text {. }
$$

As already seen in Section 3, the probability of having a connected chain composed exactly by $i+1$ vehicles is given by: $\left(1-\mathrm{e}^{-\beta R}\right)^{i} \mathrm{e}^{-\beta R}$. From this, we can calculate the average length of a multi-hop connected chain of vehicles:

$$
\begin{aligned}
\bar{L}_{\text {chain }} & =\int_{0}^{\infty} l f_{L}(l) \mathrm{d} l \\
& =\sum_{i=0}^{\infty}\left(1-\mathrm{e}^{-\beta R}\right)^{i} \mathrm{e}^{-\beta R} \bar{L}_{\text {chanin }}(i+1) .
\end{aligned}
$$




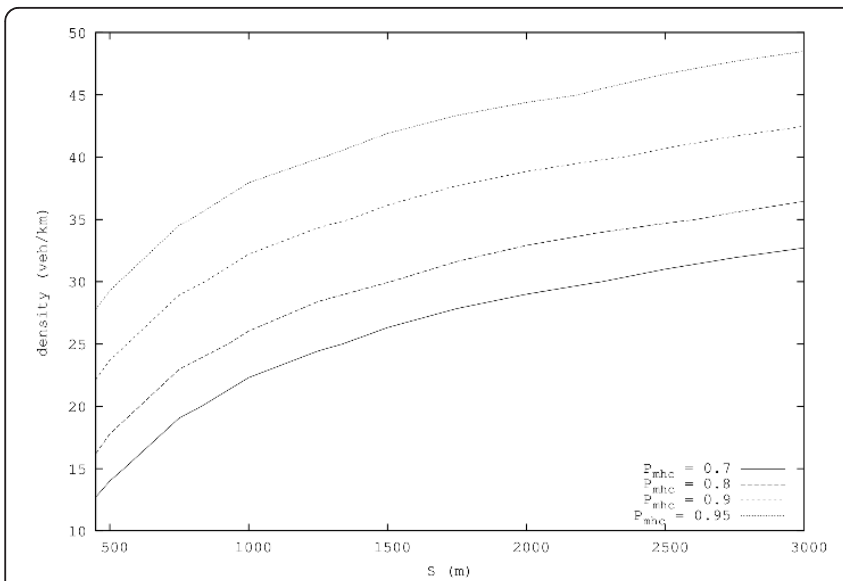

(a) $R=150 \mathrm{~m}$

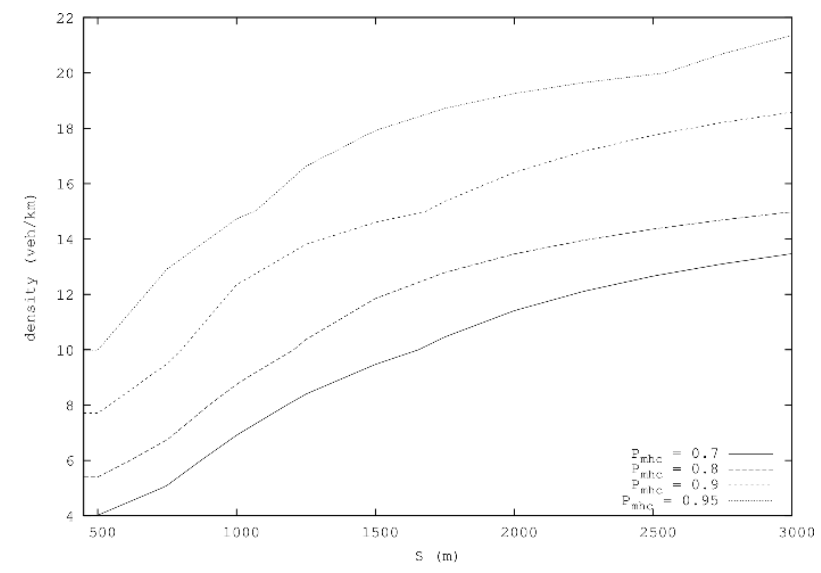

(b) $R=300 m$

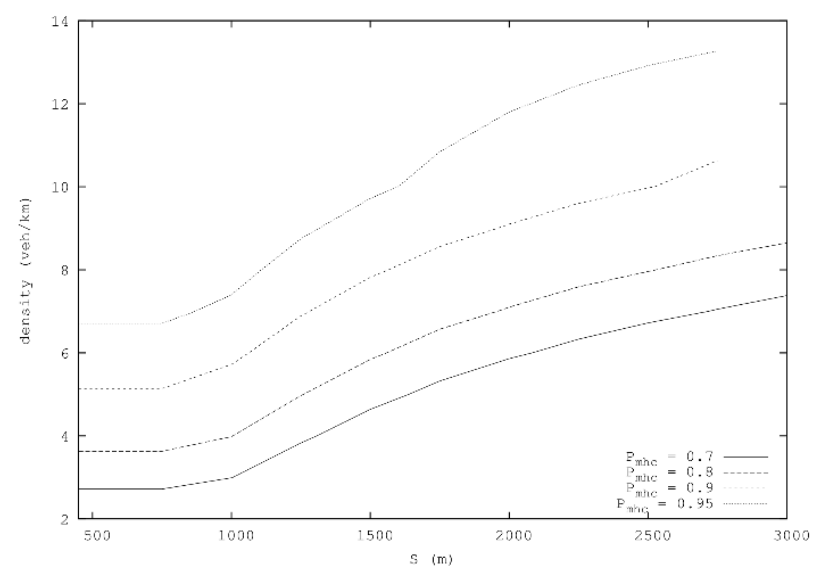

(c) $R=450 m$

Figure 6 Contours for different values of $P_{\text {mhc }}$

Therefore, the average gap length is given by:

$$
\bar{D}_{\text {gap }}=\frac{D_{\mathrm{RSU}}}{2}-R-\bar{L}_{\mathrm{chain}} .
$$

Let $T_{\mathrm{RA}}^{\mathrm{unsol}}$ be the time elapsed since a vehicle changes area until the RSU sends the next unsolicited RA. The average value of this time is given by [21]:

$$
\bar{T}_{\mathrm{RA}}^{\mathrm{unsol}}=\frac{R_{M}^{2}+R_{M} R_{m}+R_{m}^{2}}{3\left(R_{M}+R_{m}\right)} .
$$

Now we can calculate theaverage ETSI SLAAC address configuration time $\left(\bar{T}_{\text {conf }}\right)$, by simply considering the two possible configuration situations: (i) there is on average multi-hop connectivity between the unconfigured vehicle and the RSU (i.e., $\bar{D}_{\text {gap }} \leq 0$ ), and therefore vehicles only need to wait $\bar{T}_{\mathrm{RA}}^{\mathrm{unsol}}$ for the next unsolicited RA sent by the RSU; (ii) there is on average no chain of connected vehicles between the unconfigured node and the RSU:

$$
\bar{T}_{\text {conf }}= \begin{cases}\bar{T}_{\mathrm{RA}}^{\text {unsol }}, & \bar{D}_{\text {gap }} \leq 0, \\ \frac{\bar{D}_{\text {gap }}}{v}+\bar{T}_{\mathrm{RA}}^{\text {unsol }}, & \bar{D}_{\text {gap }}>0 .\end{cases}
$$

In order to validate the accuracy of our model and assess the performance of our solution, we performed the following experiments. We evaluated the configuration time $\bar{T}_{\text {conf }}$ under different traffic conditions and for different deployment configuration parameters. The traffic conditions are defined by the vehicular density $(\beta)$ and speed $(v)$, while the considered deployment configuration parameters are the distance between RSUs $\left(D_{\text {rsu }}\right)$, the radio wireless coverage of each node $(R)$, and the average time between unsolicited RAs $\left(T_{\mathrm{RA}}\right)$. The same Matlab-based simulator that was used in Section 3 to assess the effectiveness of multi-hop unicast communications in a vehicular scenario is used in these experiments. Therefore, an ideal wireless technology is assumed. In the next section we also perform an experimental evaluation based on a more complex model, and 


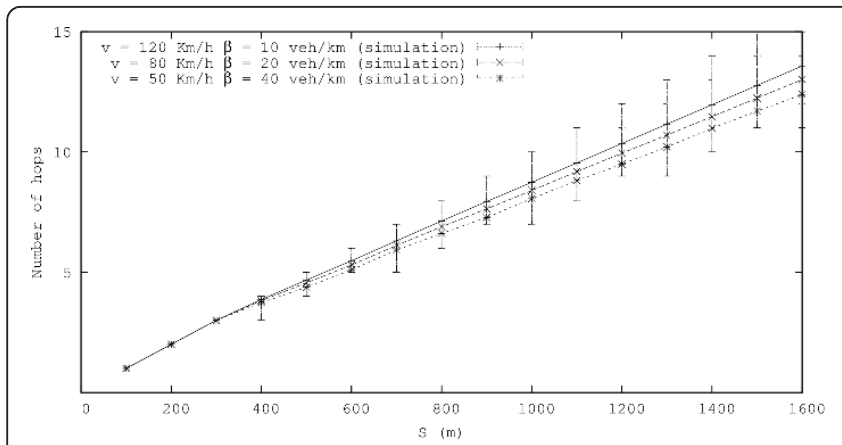

(a) $R=150 \mathrm{~m}$

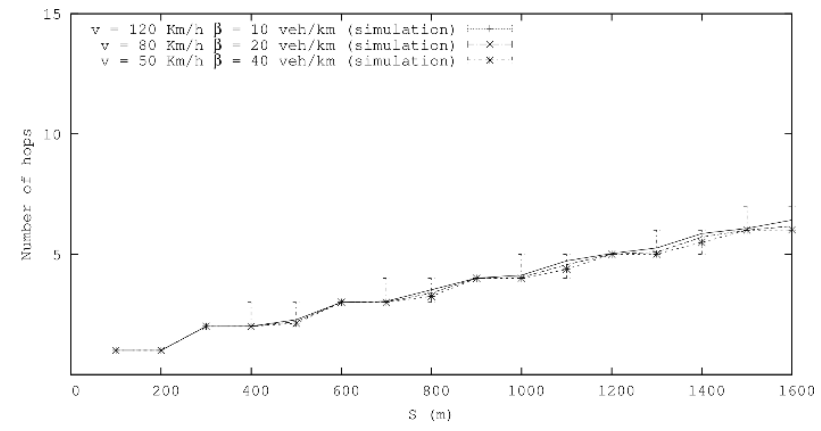

(b) $R=300 \mathrm{~m}$

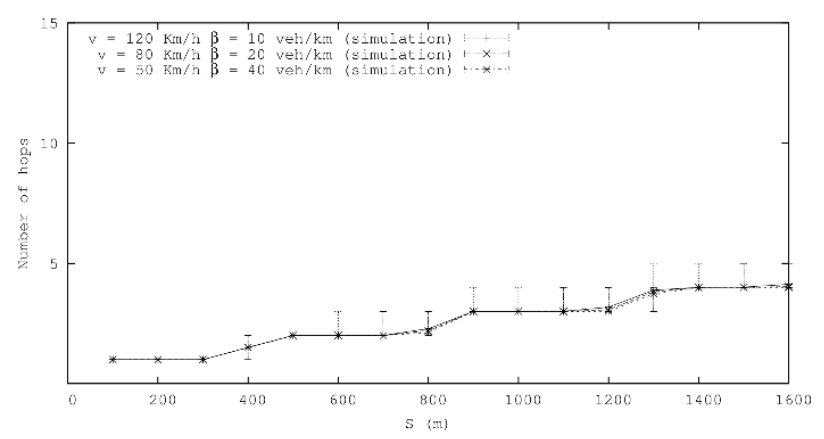

(c) $R=450 m$

Figure 7 Number of hops: simulation results

using the $\mathrm{OMNeT}++$ simulator, that allows us to assess the correctness of the simplifications assumed in our mathematical model of the ETSI SLAAC configuration time, and also to derive some configuration guidelines.

The results obtained from our simulations (with a confidence interval of 95\%) are shown in Figures 9, 10 and 11, in which the values calculated from our analysis are also depicted. We make use of the same three scenarios that we used in Section 3: urban, city highway, and motorway, and we also represent the results for different deployment scenarios (characterized by $D_{\mathrm{rsu}}$ and
$\left.T_{\mathrm{RA}}{ }^{1}\right)$. Note that the range of the average time between Router Advertisements sent by the RSU ( $T_{\mathrm{ra}}$ ) depends on the traffic conditions scenario. This is so because the maximum value that could be configured in a real scenario should allow for vehicles to always have at least one configuration opportunity before changing area and that means that $T_{\mathrm{RA}}$ has to be low enough to allow that a vehicle would be configured-in the worst possible case-when it is within one single hop of the RSU (i.e., the minimum time required by a vehicle to cross the whole coverage area of the RSU should not be higher

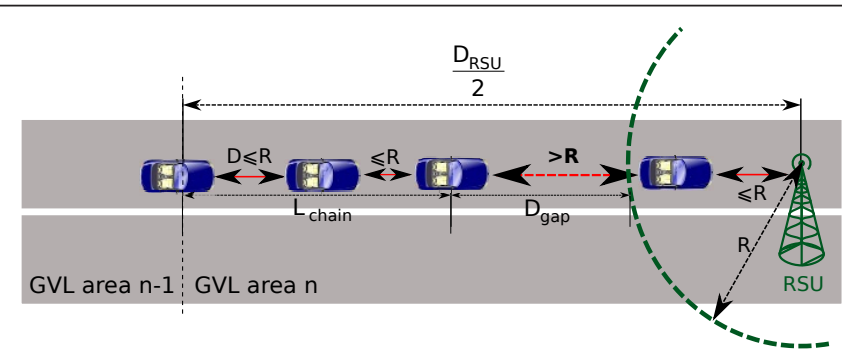

Figure 8 ETSI TC ITS IPv6 address configuration. 


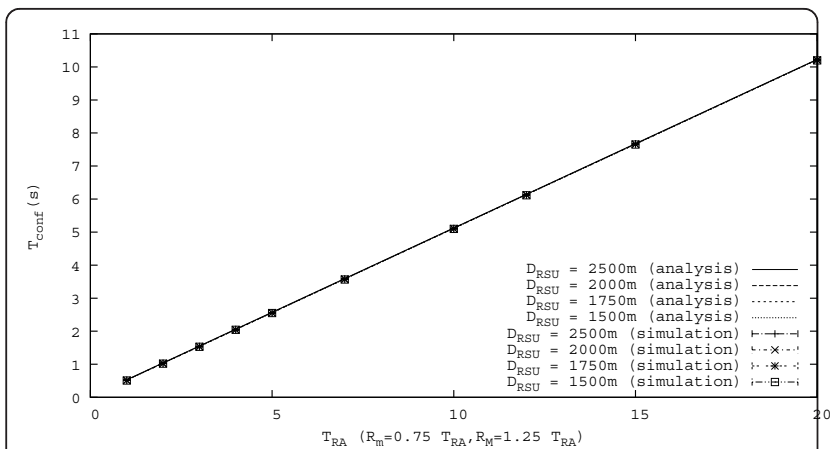

(a) $R=150 \mathrm{~m}$

Figure 9 ETSI SLAAC configuration time (analysis and simulation) for the Urban scenario.

than $R_{M}$ ). Note that in Figure 9 (urban scenario) we only show the case $R=150 \mathrm{~m}$, as the results for $R=$ 300 and $R=450 \mathrm{~m}$ are almost exactly the same (the actual difference is negligible). Similarly, for the city highway and motorway scenarios (Figures 10 and 11) we also skip (due to space constraints) depicting the results for $R=450 \mathrm{~m}$, as they are equivalent to those for $R=$ $300 \mathrm{~m}$. This is due to the fact that in the studied scenarios, the vehicular density proves to be enough to ensure multi-hop connectivity in most of the situations, and therefore $\bar{T}_{\text {conf }} \simeq \bar{T}_{\mathrm{RA}}^{\text {unsol }}$. These are reasonable scenarios in terms of vehicular density, and they are actually the ones in which it makes sense to enable Internet multihop communications, as the probability of having multihop connectivity to the RSU is high enough, and the configuration time is short enough to support classical IP communications (e.g., infotainment, non-safety). We also analyze later in the paper sparser scenarios, in which the vehicular density is much lower.

From Figures 9, 10, and 11 we can derive different conclusions. First of all, results show that our mathematical analysis perfectly matches our model of the ETSI SLAAC solution configuration time, assuming the simplifications that we have described in this section, namely: constant and homogeneous speed, perfect collision-free wireless medium, exponential inter-vehicle spacing. Another important conclusion is that in most of the scenarios, the IP address auto-configuration time can be kept very low by properly configuring the time interval between Router Advertisements, without using too aggressive values. Besides, in these scenarios the value of the wireless coverage technology $(R)$-which depends on the particular access technology, transceiver performance, antenna, and channel conditions-and the distance $D_{\text {RSU }}$ between deployed RSUs do not seem to have a noticeable impact on the resulting configuration

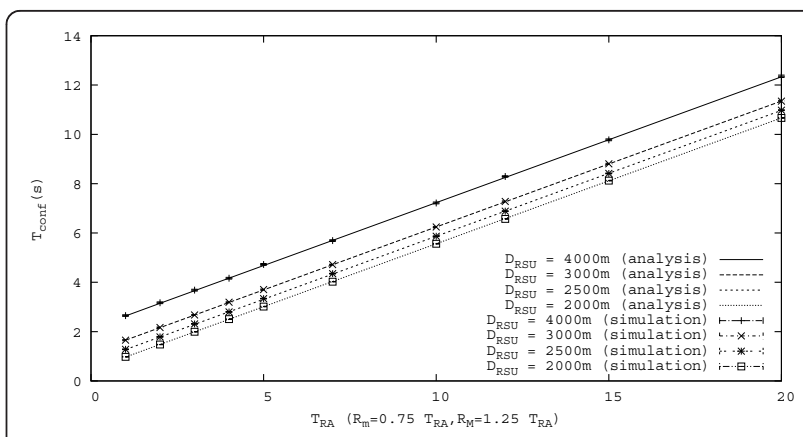

(a) $R=150 \mathrm{~m}$

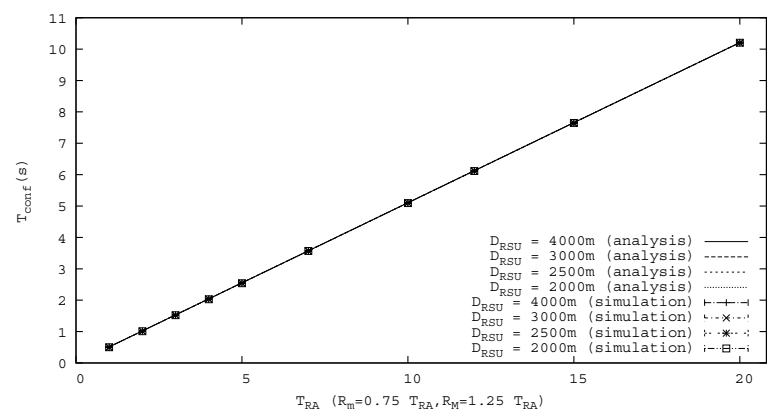

(b) $R=300 m$

Figure 10 ETSI SLAAC configuration time (analysis and simulation) for the City highway scenario.

time. Nevertheless, we should not forget that shorter values of $R$ combined with larger values of $D_{\text {RSU }}$ would lead to longer multi-hop paths, in terms of number of traversed nodes, and therefore lower effective bandwidths. Only for $R=150 \mathrm{~m}$ and in the motorway scenario (this behavior starts to be noticeable in the city highway scenario), the distance between RSUs has an impact on the configuration time, as the chances to have multi-hop connectivity between an unconfigured node that just enters into an area and the RSU decrease with the distance between them $\left(D_{\mathrm{RSU}}\right)$. In the motorway scenario, as we could anticipate from the results shown in Figure 4, configuration times are higherthough still reasonable-as the probability of having a multi-hop chain between the unconfigured node and the RSU is lower.

A simple qualitative evaluation of the ETSI SLAAC performance can be done by comparing $T_{\text {conf }}$ with the estimated permanency time of a vehicle within a geographical area. For the sake of the example, let us consider a non-extreme case, as the one of an area with a length $\left(D_{\mathrm{RSU}}\right)$ of $2,000 \mathrm{~m}$ and a wireless radio technology with 


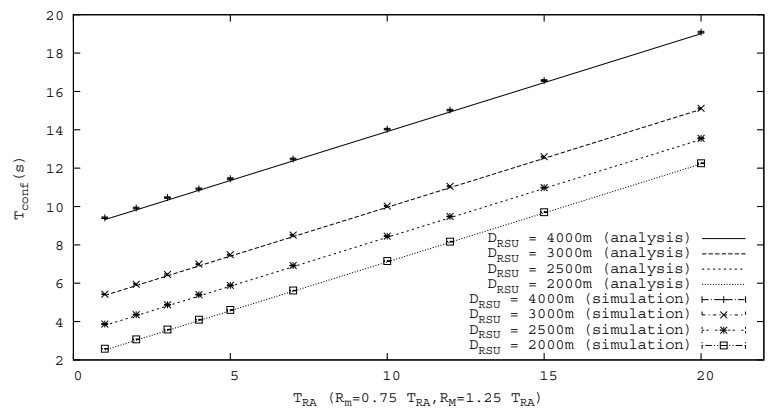

(a) $R=150 m$

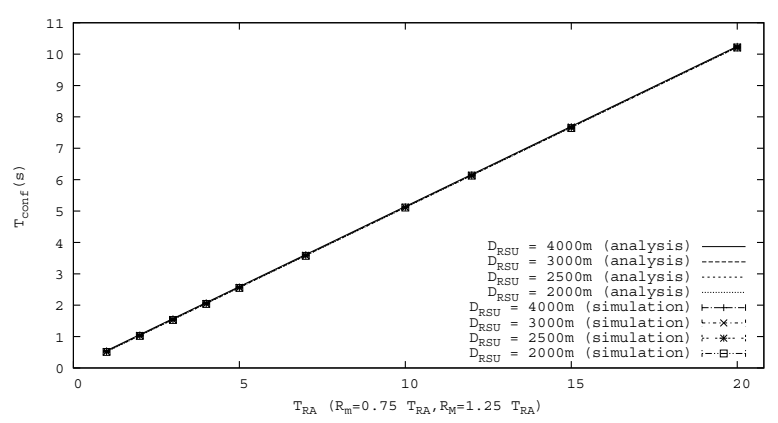

(b) $R=300 m$

Figure 11 ETSI SLAAC configuration time (analysis and simulation) for the Motorway scenario.

$R=300 \mathrm{~m}$, in the city highway scenario (average speed of $80 \mathrm{~km} / \mathrm{h}$ ). In this scenario, a vehicle spends about 90 $\mathrm{s}$ in the area. By choosing values of $T_{\mathrm{RA}}$ smaller than 10 $\mathrm{s}$, the ETSI SLAAC solution guarantees that vehicles can have Internet connectivity for more than $80 \mathrm{~s}$, as $\bar{T}_{\text {conf }}$ is approximately $5 \mathrm{~s}$. However, it is important to highlight that the configuration parameters, such as the size and shape of the geographical areas, should be chosen also taking into account the expected traffic conditions. For example, in sparse scenarios, areas should be longer than the physical radio coverage $R$, while in dense scenarios the opposite case is more appropriate. We derive some simple configuration guidelines in Section 5 .

In addition to these experiments, we performed some simulations to validate the correctness of our mathematical analysis also in scenarios in which the vehicular density is not high enough to have multi-hop connectivity during most of the time $(\beta=10 \mathrm{veh} / \mathrm{km}, v=100$ $\mathrm{km} / \mathrm{h}$ ). Examples of this scenario are city highways and motorways at night, or secondary roads. Results (see Figure 12) show that our mathematical analysis also matches the simulation results in sparse scenarios (i.e., with low values of $P_{\text {mhc }}$ ). Regarding the time required to configure a new address, obtained results confirm what we could anticipate from Figure 6, that is, in these scenarios it takes a long time to get an address, because in many cases the vehicle is not able to receive an RA until it is within the 1-hop coverage of the RSU. This also means that during a considerable amount of the time a vehicle is visiting a geographical area, it does not benefit from having connectivity with the RSU. It can also be observed that in this kind of scenario $R$ has a bigger impact on the performance, as higher values of $R$ lead to higher multi-hop connectivity probabilities.

\section{Experimental evaluation and configuration guidelines}

In this section we take a step further and experimentally evaluate the performance of the ETSI SLAAC solutionin terms of address configuration time-eliminating some of the simplifications assumed in the previous section. The goal is to assess if our mathematical model is still good enough when we use a simulation model that better replicates a real environment.

The first step is to use a realistic wireless environment, which is not collision-free and that models the different aspects of the physical and medium access control layers of IEEE 802.11. In order to do so, we implemented the ETSI SLAAC solution ${ }^{\mathrm{m}}$ using Mixim. Mixim $^{\mathrm{n}}$ is a framework for wireless ad hoc network for the $\mathrm{OMNeT}++$ simulator. $^{\circ}$ It provides the 802.11 MAC layer and many physical layer models (including the widely accepted path-loss, shadowing and large- and small-scale fading models [22-24]). The simulation scenario consists of a road segment where vehicles travel within a homogeneous flow. Vehicles' starting position is generated following an exponential distribution (speed and density are defined by the type of scenario: urban, city highway, or motorway, so the number of nodes involved in the simulation changes depending on the vehicular density). At the end of road segment, nodes enter a GVL area ( $D_{\text {RSU }}$ long) where a RSU is placed half the way $\left(D_{\mathrm{RSU}} / 2\right.$ far from the area border). Vehicles are equipped with a standard $802.11 \mathrm{~g}$ MAC layer, with a bitrate of $6 \mathrm{Mb} / \mathrm{s}$. When the simulation runs, the first vehicles are excluded from the results' recollection as they were already located inside the GVL area, but they are needed to build the multi-hop chain and let the subsequent vehicles be configured. When a node receives the first Router Advertisement after crossing the area border, its configuration time is recorded. Each simulation is run 20times using the same topology with a different seed, and for each parameter set 50 different topologies are generated. Then, the results are averaged on a population of at least $1,000 \times n$ Cars values, where 
$n$ Cars depends on the chosen vehicular density but, being the road segment length $15 \mathrm{~km}$, this value is not smaller than 150 . The parameters used in the simulations are summarized in Table 1.

The obtained simulation results are shown in Figure 13 for the three scenarios we used and for different inter-RSU distances. The results obtained from our mathematical model are also depicted in the figure. Note that since we are using a realistic wireless model, the value of $R$ is no longer a constant value. In order to select an appropriate value to be used in our mathematical model, we performed simulations with $\mathrm{OMNeT++}$ aiming at finding the average wireless coverage radius resulting from the wireless layer settings used in the simulations. The resulting value is $R=225 \mathrm{~m}$. From the results shown in Figure 13, we can observe that our mathematical model approximates quite well the experimental results obtained with $\mathrm{OMNeT}++$.

The last simulation experiment we performed to validate our mathematical analysis is the following. Using the $\mathrm{OMNeT}++$ simulator, we take the position and speed of vehicles on a real road from real traffic traces, and measure the ETSI SLAAC configuration time. The traces were taken at the 4-lane highway A6 in Spain, in the outbound direction from Madrid, and account for the traffic from 8:30 to 9:00 a.m. (which can be considered as near to rush hour). The total amount of samples is 2985. For each sample, we have a time-stamp and the speed of the vehicle. We consider that the measurement point is the border between two geographical areas and that each vehicle keeps the same speed while traversing the area. In our simulation environment, we fix the distance between two RSUs to 2,000 m. We plot in Figure 14 the results obtained from the simulation and our mathematical analysis (Equation 13). In Equation (13) we use the vehicular density calculated from the traces $(\beta=38.2 \mathrm{veh} / \mathrm{km})$ and the average speed $(v=95.11 \mathrm{~km} /$ h). As it can be observed from Figure 14, there is a small gap between the simulation results and our mathematical model, although the model still approximates the real performance. This gap is due to the fact that our model is a simplification of the real scenario, and as a result, our analysis is optimistic. Our model does not consider the non-idealities of the wireless media,

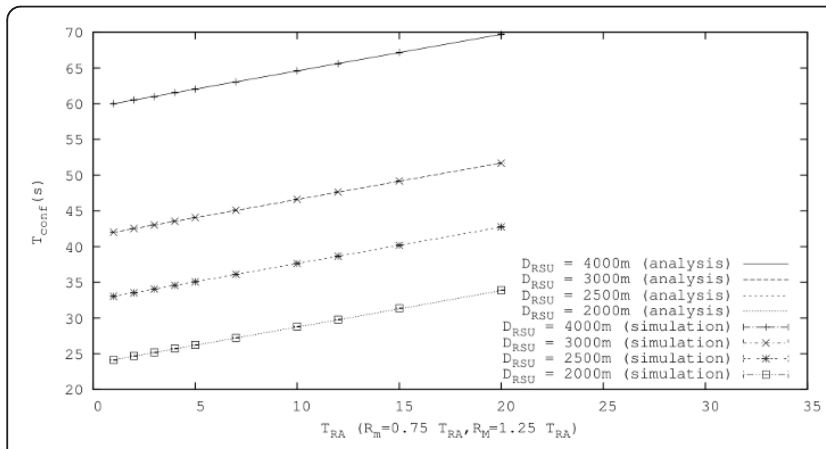

(a) $R=150 m$

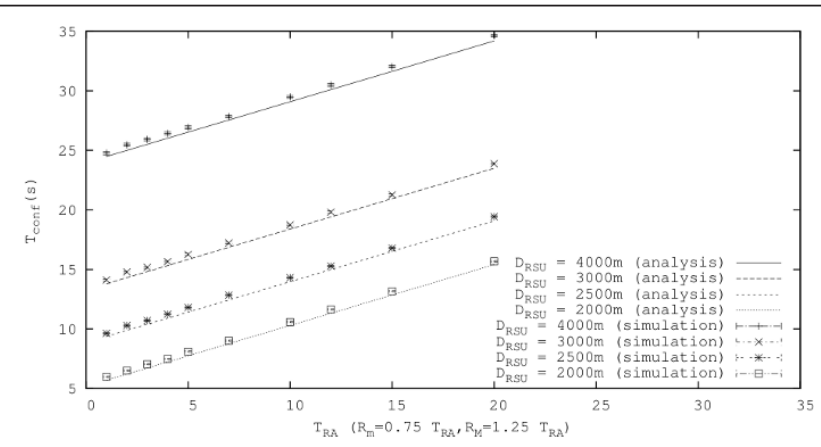

(b) $R=300 m$

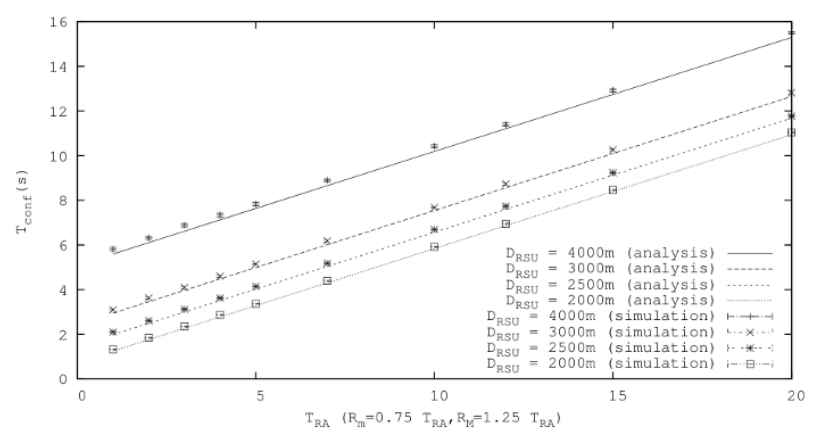

(c) $R=450 m$

Figure 12 ETSI SLAAC configuration time (analysis and simulation) for the Sparse scenario. 
assumes a perfect exponential inter-vehicle spacing and considers that all vehicles travel at the same speed. We learned from the previous experiments with OMNeT++ (Figure 13) that the deviation from considering an ideal wireless media seems to be pretty small. Therefore, we conjecture that the deviation found in Figure 14 is due to the fact that inter-vehicle spacing does not exactly follow an exponential distribution, $\mathrm{p}$ and also the fact that vehicles do not all travel at the same speed.

We have shown via simulation experiments that the ETSI SLAAC performance in terms of IP address configuration time is good enough for IP Internet-alike applications. We have also validated our mathematical model for $\bar{T}_{\text {conf }}$ and analyzed how the different deployment and configuration parameters, namely $D_{\mathrm{RSU}}, R$, and $T_{\mathrm{RA}}$, affect the obtained performance. Based on that, we provide configuration guidelines that enable the ETSI SLAAC solution to guarantee a certain performance (i.e., a target configuration time), depending on the addressed scenario. We do not include $R$ in this analysis, as this is a value that depends on the wireless technology in use, and we consider this as an external parameter that is known beforehand (all cars are equipped with the same radio technology).

- If RSUs can obtain information about the vehicular density and speed of the road segment within its assigned geographical area (this information can be obtained in real time from sensors deployed in the road, or via statistical prediction based on historical records), RSUs can dynamically calculate the minimum required $T_{\mathrm{RA}}$ to achieve the target configuration time, based on the mathematical analysis described in Section 4, namely in Equation (13). Note that we assume that RSUs can be provisioned with the location of neighboring RSUs, and therefore that they know $D_{R S} U$. The value of $T_{\mathrm{RA}}$ can then change dynamically to react and adapt to traffic conditions. In the case that RSUs do not have enough computation resources, they can be provided with a precomputed set of configuration parameters.

- If RSUs do not have any information about current vehicular density, two different configuration strategies could be followed. An optimistic approach would be based on assuming that the vehicular density is enough to safely assume that vehicles have multi-hop connectivity with the RSUs with high probability. In this case, the

Table 1 Simulation parameters

\begin{tabular}{lcc}
\hline Scenario & Speed $\mathbf{( k m} / \mathbf{h})$ & Density $(\mathbf{v e h} / \mathbf{k m})$ \\
\hline Urban & 50 & 80 \\
City highway & 80 & 50 \\
Highway & 120 & 35 \\
MAC layer & & $802.11 \mathrm{~g}$ \\
Bitrate & \multicolumn{3}{c}{$\mathbf{M b} / \mathrm{s}$} \\
\hline
\end{tabular}

RSU can make use of Equation (12). On the other hand, a more conservative approach in which no assumption can be made about the vehicular density would be based on selecting a $P_{\mathrm{mhc}}$ value that still supports reasonable levels of connectivity and makes use of the mathematical analysis described in Section 4. Note that we also assume here that RSUs are configured with $D_{\mathrm{RSU}}$.

In this case, dynamic reconfiguration of the RSU is not possible or just very limited (e.g., configuration based on the time of the day).

\section{Conclusions}

This article makes a thorough analysis of the IPv6 integration in vehicular networks, paying special attention to the IPv6 address auto-configuration functionality. We consider the recently standardized mechanisms by the ETSI TC ITS, and first assess the feasibility of IPv6 multi-hop communications, by means of analysis and simulation. Then, we focus on the IPv6 address autoconfiguration, explaining how IPv6 SLAAC mechanisms can be run over the ETSI TC ITS protocol stack, and characterizing the configuration time performance.

Multi-hop vehicular networks are needed to reduce the cost of fix infrastructure deployment. This article explores which are reasonable scenarios for Internet access from vehicles, considering vehicular density, the radius of coverage of the wireless technology, and the distance between access points in the infrastructure. The reasonable scenarios are characterized by a high probability of reaching the fixed infrastructure from vehicles. These are the target scenarios for address autoconfiguration solutions for vehicular networks. We make the point that considering other scenarios blindly can lead to misleading results about the performance of the solutions, since when communications are not possible, a success of failure in configuring an address is basically irrelevant.

In the article we derive an analytical expression for the average time interval required by a vehicle using the ETSI SLAAC solution to configure a new address; and we validate the analytical model by means of extensive simulations using realistic wireless transmission model and real vehicular traces. Finally, the article also provides configuration guidelines that allow to make designs for achieving target address configuration delays in different scenarios. In terms of solution performance, particular focus is put on this address configuration time interval, which is a key figure of merit due to the possibly frequent subnet changes experienced by a moving vehicle. The detailed analytical characterization of the ETSI SLAAC solution allows to obtain pre-determined performance upper limits defined by the applications, facilitating a gradual and effective deployment of short-range Internet-based vehicular applications. The 


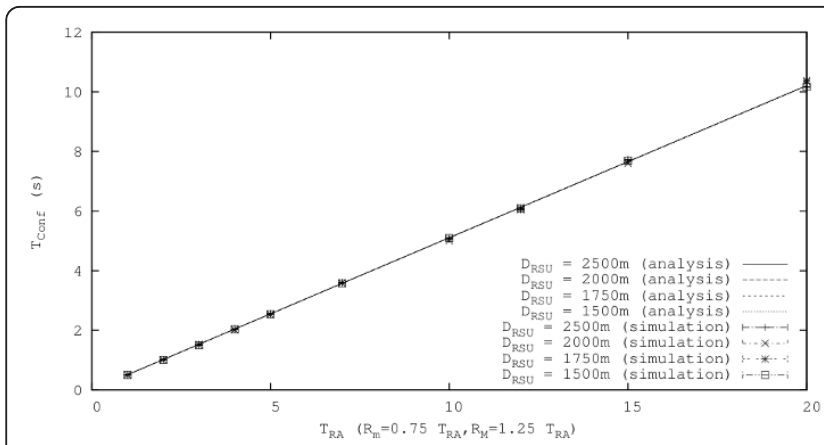

(a) Urban road scenario

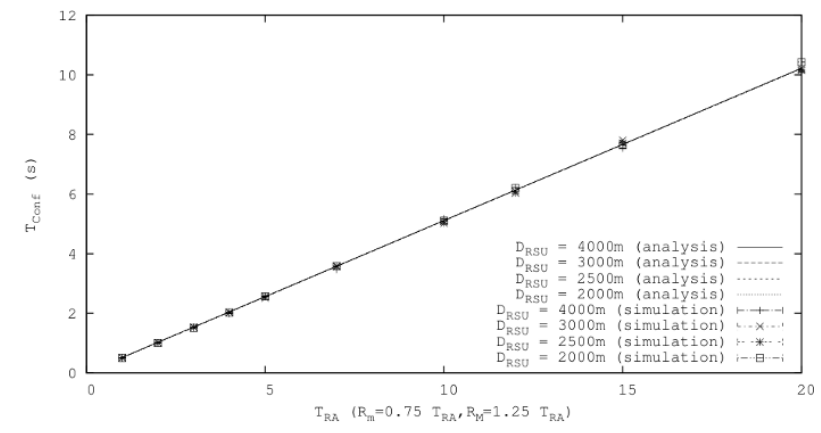

(b) City highway scenario

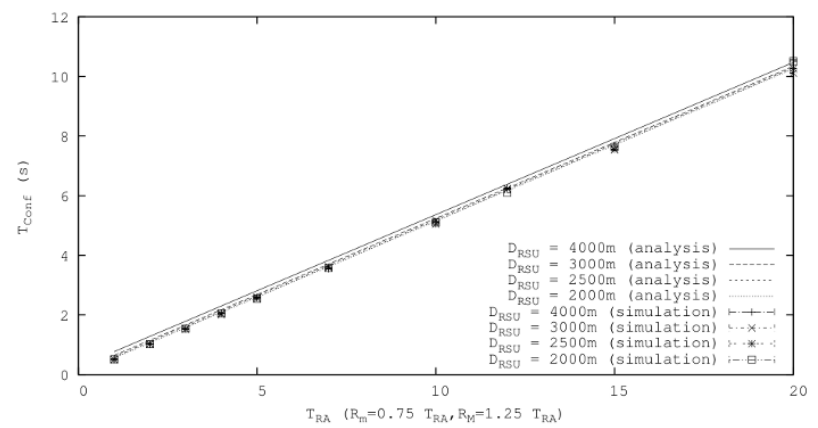

(c) Motorway scenario

Figure 13 ETSI SLAAC configuration time (analysis and simulation with OMNeT++).

obtained results of our analysis show that there is room for additional extensions to the base ETSI SLAAC solution, which can reduce the IP address configuration time by introducing additional complexity (e.g., in terms of changes to the IPv6 stack or additional processing). Among the several approaches that are worth exploring, we can mention for example the use of maps with prefix

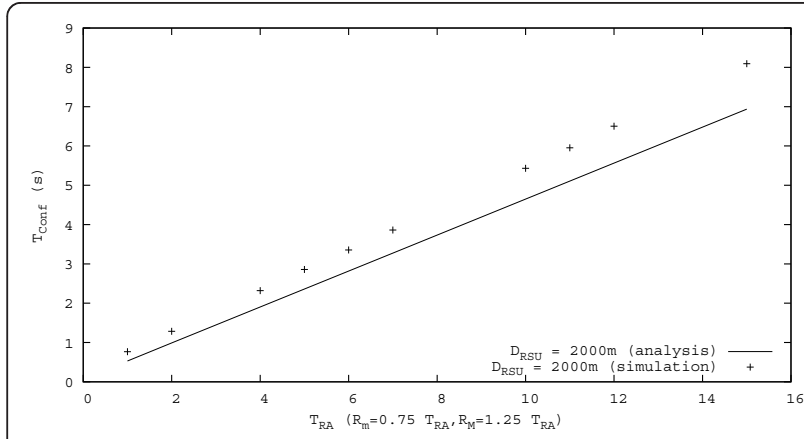

Figure 14 ETSI SLAAC configuration time (analysis and simulation with OMNeT++ and real traffic traces). information enabling ITS stations to know in advance the advertised IPv6 prefixes per GVL area, or the overhearing of prefix information of neighboring GVL areas [25]. There might be applicability scenarios that deserve a careful analysis of the trade-offs involved by implementing extensions to the base ETSI SLAAC solution.

Next steps include the implementation of the ETSI TC ITS architecture and its validation and performance evaluation in real environments, for example by means of field operational tests (FOTs) conducted in the testbed platforms currently being deployed in Europe.

\section{Endnotes}

ahttp://www.standards.its.dot.gov/fact_sheet.asp?f=80

${ }^{b}$ http://www.iso.org/iso/iso_technical_committee? commid $=54706$

${ }^{c}$ http://www.etsi.org/website/Technologies/IntelligentTransportSystems.aspx

${ }^{\mathrm{d}}$ http://www.car-to-car.org/.

e Internet Engineering Task Force: http://www.ietf.org/.

f For example, 2001:DB8:1:1::/64 and 2001: DB8:1:2::/64 are non-overlapping prefixes, while 
$2001:$ DB8 : $1:: / 48$ and $2001:$ DB $8: 1: 2:: / 64$ are overlapping..

ghttp://www.geonet-project.eu/.

${ }^{\mathrm{h}}$ More precisely, in this solution gateway selection is performed by the infrastructure itself and not by the nodes as in many MANET approaches..

${ }^{\mathrm{i}}$ The code of this simulator is available at http://fourier.networks.imdea.org/people/ marco_gramaglia/sims/ ETSI-SLAAC/

${ }^{j}$ It is also very likely that in the future, cars are equipped with a dedicated interface for safety-related communications and one (or several, but of different technologies) for IP non-safety-related communications..

${ }^{\mathrm{k}} \mathrm{We}$ consider the speed of all vehicles fixed and constant for simplicity of the model. Simulation results that we will present later show that this simplification does not affect the validity of the conclusion of our analysis..

${ }^{\mathrm{l}} R_{M}=1.25 T_{\mathrm{RA}}$ and $R_{m}=0.75 T_{\mathrm{RA}} \cdot$

${ }^{\mathrm{m}}$ The code of this simulator is available at http://fourier.networks.imdea.org/people/ marco_gramaglia/sims/ ETSI-SLAAC/

${ }^{n}$ http://mixim.sourceforge.net/.

ohttp://www.omnetpp.org/.

PWe analyzed the inter-vehicle spacing from the used traces and it is close to an exponential distribution, as suggested in [15], but with some minor deviation. As future work, we are currently modeling this inter-vehicle spacing, based on real traffic traces [26].

\section{Acknowledgements}

The authors would like to acknowledge the Spanish Directorate General of Traffic for kindly providing us with the empirical traces used in this work. The research of Marco Gramaglia and Carlos J. Bernar-dos leading to these results has received funding from the European Community's Seventh Framework Programme (FP7-ICT-2009-5) under Grant agreement no. 258053 (MEDIEVAL project). The research of Marco Gramaglia, Carlos J. Bernardos and Maria Calderon has also received funding from the Ministry of Science and Innovation of Spain, under the QUARTET Project (TIN2009-13992-C02-01).

\section{Author details}

${ }^{1}$ Institute IMDEA Networks, Madrid, Spain ${ }^{2}$ Universidad Carlos III de Madrid, Madrid, Spain ${ }^{3}$ Universidad Politécnica de Madrid, Madrid, Spain ${ }^{4}$ NEC Laboratories, Network Division, NEC Europe Ltd, Heidelberg, Germany

\section{Competing interests}

The authors declare that they have no competing interests.

Received: 1 June 2011 Accepted: 17 January 2012

Published: 17 January 2012

\section{References}

1. ETSI, Intelligent Transport Systems (ITS), Vehicular Communications; Part 6: Internet Integration; Sub-Part 1: Transmission of IPv6 Packets over GeoNetworking Protocols. ETSI TS 102 636-6-1 V1.1.1. (March 2011)

2. ETSI, Intelligent Transport Systems (ITS); Vehicular Communications; GeoNetworking; Part 3: Network Architecture. ETSI TS 102 636-3 V1.1.1. (March 2010)

3. T Narten, E Nordmark, W Simpson, H Soliman, Neighbor Discovery for IP version 6 (IPv6). RFC 4861. (September 2007)

4. S Thomson, T Narten, T Jinmei, IPv6 Stateless Address Autoconfiguration. RFC 4862. (September 2007)
5. R Droms, J Bound, B Volz, T Lemon, C Perkins, M Carney, Dynamic Host Configuration Protocol for IPv6 (DHCPv6). RFC 3315 (July 2003)

6. CJ Bernardos, M Calderon, H Moustafa, Survey of IP Address AutoConfiguration Mechanisms for MANETs. IETF, draft-bernardosmanetautoconf-survey-05.txt (work-in-progress). (June 2010)

7. M Fazio, C Palazzi, S Das, M Gerla, in Proceedings of the 1st IEEE Workshop on Automotive Networking and Applications (AutoNet). Vehicular Address Configuration. GLOBECOM 2006 (San Francisco, CA, USA, 2006)

8. R Baldessari, CJ Bernardos, M Calderon, in PIMRC 2008, GeoSAC-Scalable Address Au-toconfiguration for VANET Using Geographic Networking Concepts (Cannes (France), September 2008)

9. J Choi, Y Khaled, M Tsukada, T Ernst, in The 8th International Conference on Intelligent Transport System Telecommunications (ITST 2008), IPV6 Support for VANET with Geographical Routing (October 2008)

10. V Devarapalli, R Wakikawa, A Petrescu, P Thu-bert, Network Mobility (NEMO) Basic Support Protocol. RFC. 3963 (January 2005)

11. ETSI, Intelligent Transport Systems (ITS); Vehicular Communications, Part 4: Geographical Addressing and Forwarding for Point-to-Point and Point-toMultipoint Communications; Sub-Part 1: Media-Independent Functionality. ETSI TS 102 636-4-1 (work in progress). (May 2011)

12. J Ott, F Kutscher, in Proceedings VTC Fall The Drive-Thru Architecture: WLAN-Based Internet Access on the Road. (May 2004)

13. E Fonseca, A Festag, R Baldessari, R Aguiar, in Proceedings of IEEE Wireless Communications and Networking Conference (WCNC). Support of Anonymity in VANETs-Putting Pseudonymity Into Practice (Hong Kong, March 2007)

14. C Harsch, A Festag, P Papadimitratos, in Proceedings VTC Fall, Secure Position-Based Routing for VANETs (Baltimore, MD, USA, 2007)

15. H Reijmers, R Prasad, in 48th IEEE Vehicular Technology Conference, 1998. VTC 98. The Influence of Vehicle Distribution Models on Packet Success Probability on a Three Lane Motorway, 3 (1998)

16. N Wisitpongphan, F Bai, P Mudalige, V Sadekar, O Tonguz, Routing in Sparse Vehicular Ad Hoc Wireless Networks. IEEE J Sel Areas Commun. 25(8), 1538-1555 (2007)

17. L Bain, D Weeks, A note on the truncated exponential distribution. Ann Math Stat. 35(3), 1366-1367 (1964). doi:10.1214/aoms/1177703298

18. R Haberman, Mathematical models: mechanical vibrations, populations dynamics and traffic flows : an introduction to applied mathematics, SIAM Soc Ind Appl Math, Philadelphia, PA (1998)

19. A Festag, R Baldessari, H Wang, in Proceedings of 4th International Workshop on Intelligent Transportation (WIT), On Power-Aware Greedy Forwarding in Highway Scenarios (Hamburg, Germany, 2007), pp. 31-36

20. D Johnson, C Perkins, J Arkko, Mobility Support in IPv6. RFC 3775. (June 2004)

21. YH Han, J Choi, SH Hwang, Reactive Handover Optimization in IPv6-Based Mobile Networks. IEEE J Sel Areas Commun. 24(9), 1758-1772 (2006)

22. A Köpke, M Swigulski, K Wessel, D Willkomm, PTK Haneveld, TEV Parker, OW Visser, HS Lichte, S Valentin, in Simutools '08: Proceedings of the 1st International Conference on Simulation Tools and Techniques for Communications, Networks and Systems \& Workshops. Simulating Wireless and Mobile Networks in omnet++ the Mixim Vision, 1-8 (2008)

23. JK Cavers, Mobile Channel Characteristics (Kluwer, Dordrecht, 2000)

24. MK Simon, MS Alouini, Digital communications over fading channels (m.k. simon and m.s. alouini; 2005) [book review]. IEEE Trans Inf Theory 54(7), 3369-3370 (2008)

25. M Gramaglia, I Soto, CJ Bernardos, M Calderon, Overhearing assisted optimization of address auto-configuration in position aware VANETs. IEEE Trans Veicular Technol. 60(7), 3332-3349 (2011)

26. M Gramaglia, P Serrano, JA Hernandez, M Calderon, CJ Bernardos, in Proceedings of IEEE International Symposium on a World of Wireless, Mobile and Multimedia Networks (WoWMoM), New insights from the analysis of free flow vehicular traffic in highways (Lucca, Italy, 2011)

doi:10.1186/1687-1499-2012-19

Cite this article as: Gramaglia et al:: IPv6 address autoconfiguration in geonetworking-enabled VANETs: characterization and evaluation of the ETSI solution. EURASIP Journal on Wireless Communications and Networking 2012 2012:19. 